BAPIOAC19-P06

\title{
Assessment of bowel motion in patients receiving pelvic Stereotactic ablative radiotherapy (SABR)
}

Poster presented at BAPIO National Conference, London 2019 Peer reviewed by Subarna Chakravorty PhD \& Sunil Daga PhD

\author{
Isobel Rycroft \\ Cite as: Rycroft, I. (2020) Assessment of \\ bowel motion in patients receiving pelvic \\ Stereotactic ablative radiotherapy (SABR). The \\ Physician vol 6; issue 6; epub 01 Jan 2020; DOI: \\ https://doi.org/10.38192/1.6.2.11 \\ Article Information \\ Submitted 01 Nov 2019 \\ Published 01 Aug 2020 \\ Open Access - Creative Commons Licence CC-BY- \\ ND-4.0
}

\section{Background:}

Stereotactic ablative body radiotherapy (SABR) is a novel technique that delivers high radiotherapy doses and is used to treat pelvic oligometastatic nodal disease. Pelvic radiotherapy can damage bowel. As a mobile structure, bowel position can change. It is unknown to what extent bowel mobility changes the dose it receives in SABR treatment. This study aimed to investigate the impact of bowel mobility on the dose it receives between and during (SABR) treatments.

\section{Methods:}

Planning CT plus pre and post-treatment Cone Beam CT (CBCT), for each fraction of treatment, were acquired for 5 patients. Bowel within a $3 \mathrm{~cm}$ margin around the planning target volume (PTV) was contoured on all CBCTs and contours were superimposed onto the planning $\mathrm{CT}$, to allow volumetric and dosimetric assessment. The volume of bowel within the $3 \mathrm{~cm}$ margin, nearest edge of bowel to the PTV, mean dose and the maximum dose to $0.5 \mathrm{~cm} 2$ were recorded. Measures for pre and post- treatment $\mathrm{CBCTs}$ were compared to determine motion during one treatment (intra-fraction). Measures for pretreatment CBCTs and planning CT were compared to determine motion between treatments (inter-fraction). Wilcoxon signed-ranks tests were used for comparisons.

\section{Results:}

No significant differences in inter- or intra- fraction motion were noted for the whole population. However, for individual patients, bowel positions showed considerable variation and clinically relevant dosimetric changes were noted between and during treatment.

\section{Conclusions:}

Individual CBCT images should be reviewed for individual patients receiving pelvic SABR as in some cases the bowel may receive higher doses than intended.

SABR 